\title{
SMART METERING OF RENEWABLE MICRO GENERATORS BY OUTPUT PATTERN RECOGNITION
}

\author{
Peter BOAIT \\ De Montfort University - UK \\ pboait@dmu.ac.uk
}

\begin{abstract}
Micro generators need to be registered and metered when connected to the distribution network, and the energy source for renewable generation needs to be certified. Pattern recognition techniques are described which allow these functions to be performed automatically as part of a smart metering system. Potential benefits are reduced costs for Distribution Network Operators, simplified installation procedures for micro generator suppliers, and for users improved performance information and access to the financial incentives for renewable generation.
\end{abstract}

\section{INTRODUCTION}

Government policy in most countries seeks to encourage the installation of micro generators that can capture energy from renewable sources, or make more efficient use of fuels through the production of Combined Heat and Power (CHP). Often there are financial incentives for renewable sources, such as feed-in tariffs (as in Germany) or Renewable Obligation Certificates (UK), while CHP may attract lower incentives or none. These incentives carry with them the need for registration of the capacity and energy source of each micro generator, and then metering of the generator output. However, to motivate as many home owners and small businesses as possible to adopt these technologies the installation process needs to be as simple as possible. Ideally the purchaser of a micro generator should be able to "plug and play" subject to compliance with essential safety standards. The Distribution Network Operator (DNO) will also benefit from reduced costs if this can be achieved.

The introduction of "smart" meters of various forms presents the opportunity to automate registration and metering processes for renewable micro generators. This paper describes methods for:

- recognising the presence of a micro generator,

- identifying the energy source and type of generator plant, and

- characterising the generator output,

using time and frequency domain pattern recognition techniques that can be readily integrated into a metering system with data processing capability. It will be shown that the energy source for any micro generator can be identified from time and frequency domain analysis of the variations in output power. It is also possible to discriminate the contributions from different energy sources in a single aggregated power flow, allowing the energy from renewable and non renewable sources to be separately metered from a single measurement point. The discussion focuses on photovoltaic (PV), wind, and CHP generators since these are the three types that are readily available at the micro scale $(<5 \mathrm{~kW}$ rated capacity) and are being installed in homes and business premises in increasing numbers.

\section{MICRO GENERATOR CONNECTION}

The methods assume that any generator is connected to the mains supply terminals in a manner similar to the UK standard G83/1 [1] as shown in Figure 1. The metering system is equipped with an additional sensor that allows it to separately measure the power $P_{1}$ drawn from the mains supply and power $P_{2}$ flowing into the electrical load at the premises. Negative values for $P_{l}$ are taken to imply export. The total power from one or more local generators is given by $P_{3}=P_{2}-P_{1}$. The time series for $P_{3}$ can then be subjected to time and frequency domain analysis to identify the properties of each generator contributing to it.

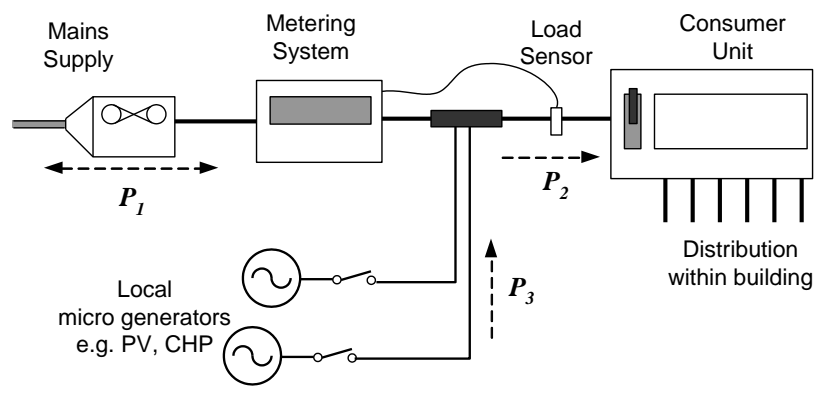

Figure 1. Micro generator connection schematic

It is assumed that the metering system has some form of telecommunications capability with the supplying utility allowing the generator characterisation and metering data to be transmitted and acknowledged. 


\section{PATTERN RECOGNITION METHODS}

The time varying profile of $P_{3}$ is characteristic of the type of generator. For example, the output of a PV generator has an upper limit set by the pattern of solar radiation. By summing the value of $P_{3}$ for each time of day over a period in the range 7-30 days the metering system can obtain a mean daily profile for $P_{3}$ which does not contain random minute-to-minute variations. The mean profile obtained from a $1 \mathrm{kWp}$ PV generator for June is shown in Figure 2, compared to a single day. This can be readily matched to the expected bell-shaped curve for solar radiation.

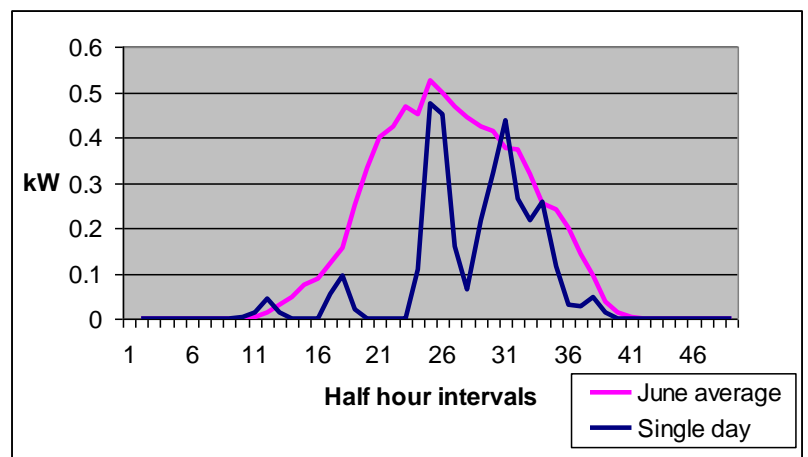

Figure 2. Single day and monthly average output from a PV generator

A micro wind generator has the distinguishing feature of producing some energy over the entire 24 hour cycle when averaged over a suitable period. However, the average profile for a micro CHP generator is less obviously recognisable, as shown in Figure 3. The micro CHP in question had a peak output of $0.95 \mathrm{~kW}$ and was just heating domestic hot water in the month plotted - a different household might have fewer or more instances of hot water use.

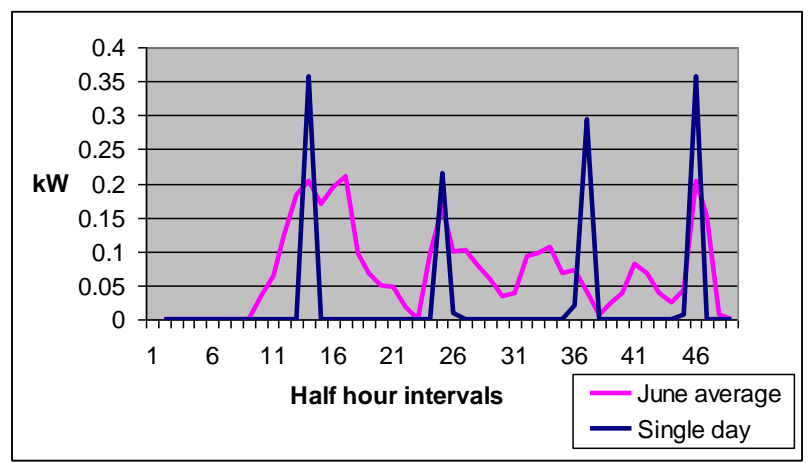

Figure 3. Single day and monthly average output from a micro CHP generator

To improve the robustness of pattern matching, a frequency domain comparison can be employed as well as time domain. A Fourier series representation of any periodic function is possible provided that:

$$
\int f(t) d t
$$

is finite over a complete period and is continuous. If these conditions are satisfied the function can be represented as:

$$
f(t)=a_{0}+\sum_{n=1}^{\infty} a_{n} \cos \frac{2 \pi n t}{T}+\sum_{n=1}^{\infty} b_{n} \sin \frac{2 \pi n t}{T}
$$

The periodicity on a daily cycle of a PV generator is self evident, while that of a micro CHP arises from the dependence of heat demand on the occupancy cycles of a home or business. Wind has periodicities at several timescales from daily to seasonal - this has been shown by Sinden [2] and others. A tidal generator would clearly have a daily or twice daily periodicity. A small hydro generator may not show periodicity so is probably not amenable to this technique.

Figures 4 and 5 below show the results of applying a Fourier transform to a 30-day series of PV and micro CHP energy output data for January and May. This resolves the $a_{n}$ and $b_{n}$ vectors at each frequency into a single value. The $\mathrm{Y}$ axis of a Fourier transform would normally show energy spectral density values - in this case Joules per frequency interval for half of the energy in the series because the other half is translated into negative frequency values that are not shown in a conventional one-sided plot. Also the series is infinite so some of the energy is not shown. So to simplify the presentation the $\mathrm{Y}$ axis in Figures 4 and 5 has been normalised by taking the first frequency axis point for each month (the value for $0-2$ cycles/month) as unity.

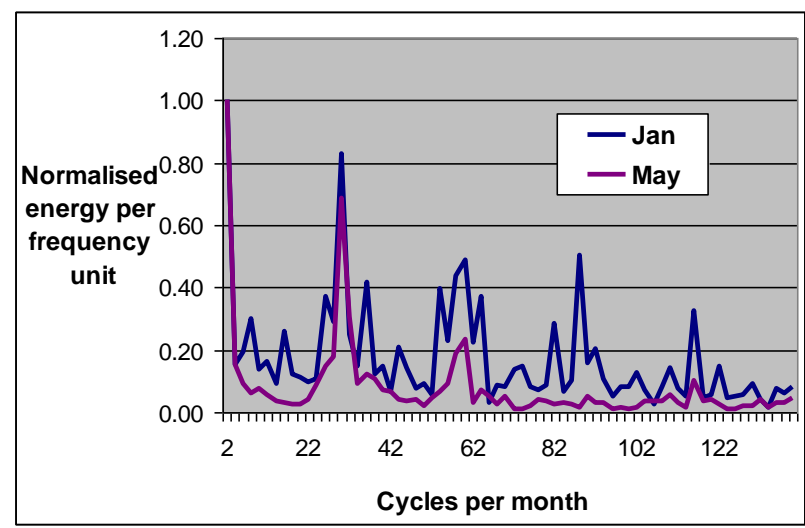

Figure 4. Fourier transform of the output series from a PV generator

The consistency in the patterns for each generator type can be seen even though their energy outputs in the months shown are very different. The most prominent distinguishing feature is the level of the second peak which shows the energy at a daily periodicity - for PV it is about 0.8 whereas for micro CHP it is 0.25 . Also the twice daily third peak for micro CHP is more or less equal to the daily 
peak whereas for PV it is much reduced. A wind generator has a broad first peak because the cyclical pattern of weather fronts lasting several days increases the energy in the $0-10$ cycles/month region of the spectrum. These spectral characteristics are reliable even in the presence of "noise", as visible from the intermittent nature of PV output in January or micro CHP in May.

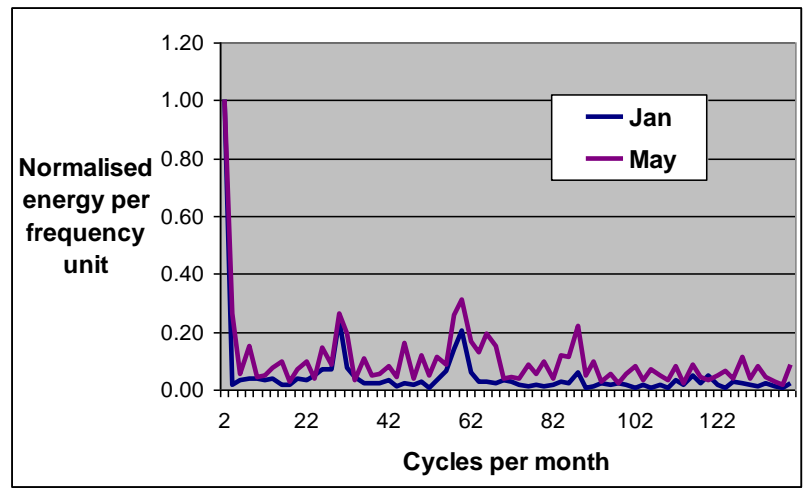

Figure 5. Fourier transform of the output series from a micro CHP generator

From this time and frequency analysis it is possible to determine whether $P_{3}$ is generated by a PV, wind, or CHP generator, or any combination of these three options, using a technique known as a "naïve Bayes classifier". This discriminates members of classes $C_{l} \ldots C_{j}$ depending on the discrete values of a set of attributes $F_{1} \ldots \ldots F_{n}$ that describe the objects to be classified. As long as $F_{1} \ldots \ldots F_{n}$ are conditionally independent it is possible to write Bayes' theorem as:

$$
p\left(C_{j} \mid F_{1} \ldots F_{n}\right)=\frac{1}{k} p\left(C_{j}\right) \prod_{i=1}^{n} p\left(F_{i} \mid C_{j}\right)
$$

i.e. the probability that a set of attribute values $F_{1} \ldots \ldots F_{n}$ describe a member of class $C_{j}$ is the product of the probability of that class occurring with the probabilities of each of the attribute values occurring given $C_{j}$, divided by a constant $\mathrm{k}$ which corresponds to the denominator $p\left(F_{1} \ldots . . F_{n}\right)$ of Bayes' theorem. This constant does not have to be evaluated when the objective is simply to find the most probable class. The method is known to work well even when there is some dependency between attributes [3].

In this case there are 7 classes comprising the three generator types occurring individually, plus the three combinations of pairs, and the class where all three are present. The probability of each class will be known from the national statistics of existing micro generator installations. A set of attributes that can be employed by a practical classifier is:
- the proportion of energy generated outside daylight hours (a non-zero value excludes the PV-only class)

- presence/absence of a mid-day peak indicating PV generation, or a mid afternoon peak indicating wind

- distribution of energy over 24 hours (the flatter the distribution the greater the wind element)

- height of the second and third spectrum peaks

- width of the first spectrum peak.

The measured value of each attribute is mapped to one of a few discrete values - for example the height of the second peak may be identified as either above 0.7 , between 0.3 and 0.7 , or below 0.3 . Each discrete value is associated with a probability for each class derived from historic data. The class probabilities can then be calculated for the set of attribute measurements and the most probable taken as the identified generator configuration.

To complete automatic registration of a generator, the peak output is needed. This can be readily measured as the maximum value of $P_{3}$ for the single generator classes, and as the dark hours peak for pairings with PV. For the other combinations an initial estimate can be obtained from the height of the spectrum peaks, which will indicate the relative proportions of the energy generated from each of the sources present. This can then be refined when one of the generators is identified as "missing" by the classifier. For example, anticyclone weather can result in several days with no wind that will allow a CHP and wind generator combination to be resolved.

Clearly reliable results will not be obtained from these methods until at least a month's data has been collected and analysed. This is not inconsistent with current manual methods of registration - G83/1 [1] allows 30 days for installers to submit paperwork. A precise measurement of maximum output from each generator in an installation with more than one type could take up to a year, so the transactions between the smart meter and the registration database will have to include measurement updates.

\section{APPLICATIONS AND BENEFITS}

It is envisaged that the techniques described above would be implemented as software applications running on a metering system in addition to the basic energy measurement and user information functions that are the motivation for smart meter rollout programmes in many countries. Although they appear complex, their computation is not time critical so can be performed as a background task by any microprocessor that is capable of handling real-time telecommunications and energy measurement. The added value that could thereby be obtained by DNOs, energy suppliers, and consumers, is summarised below. 


\section{$\underline{\text { Automatic registration }}$}

As the numbers of micro generators grow, the maintenance of records by DNOs covering their life cycle of installation, amendment, and cessation, will become increasingly onerous. A completely automatic process that tracks the micro generator population will control these costs and allow network planning and engineering to be performed with up to date and accurate data.

\section{Renewables certification and fraud prevention}

Where a financial incentive is offered for renewable generation, such as Renewable Obligation Certificates (ROCs) or premium feed in tariffs, there is clearly a need to assure that the electricity generated is from a genuine renewable source. These methods allow the source to be validated with a high confidence, and will deter or detect any attempt to obtain the financial benefits of renewables by simulating a generator using an ordinary mains supply.

Automation of the UK ROC registration and certification process for micro generators [4] would be particularly beneficial, since it is currently very complex and completely unsuitable for "mass market" use by ordinary consumers.

\section{Renewables Metering}

If all generation producing $P_{3}$ is from $\mathrm{PV}$, wind, or a combination of the two sources, then ROCs can be claimed with metering accuracy compliant with [4] and [5] i.e. within $+2.5 \%$ and $-3.5 \%$. Where both renewable and CHP micro generators are present, the separate metering of the renewable element to this level of accuracy may not be consistently achievable using these methods. A widening of the tolerance to, say, $+5 \%$ and $-10 \%$ can be argued to be a reasonable concession for micro generators given that:

- a tolerance of $10 \%$ is allowed to large power stations performing cofiring of biomass with fossil fuels in calculating the proportion of their output qualifying for ROCs [6]

- [4] specifies that the number of ROCs that can be claimed annually by micro generators is determined by rounding their output to the nearest MWh.

\section{Simplified installation with integrated display}

Any smart meter installation including a load sensor as shown in Figure 1 will be in effect "generator ready" as no additional metering will be required. There will be no need to set up a local network connection linking a separate generator meter to the smart meter for collection and onward transmission of generator meter records.

Smart meter installations generally include a user display which shows electricity consumption and cost. The ability of such a display to motivate more efficient use of energy is well established by evidence such as [7] and is probably the most important factor driving smart meter rollouts. If the display can show data on any local generation it will ensure that the user has a single source of information concerning their energy flows, allowing them to make best use of their locally generated electricity. This also represents a further cost saving for micro generator installations which at present normally provide their own display of generator power and energy output.

\section{CONCLUSIONS}

Widespread adoption of micro generators is essential to the success of policy initiatives like the UK's requirement for all new homes to be "zero carbon" by 2016 [8]. This means that the cost and complexity of their installation and management must be driven down. An analogy is the rollout of broadband internet access, which has been progressively simplified by the telecommunications industry. These techniques can contribute to this important objective.

\section{REFERENCES}

[1] ENA, 2003, Recommendations for connection of small-scale embedded generators in parallel with public low-voltage distribution networks, Energy Networks Association, London, UK, 12-16.

[2] G. Sinden, 2007, "Characteristics of the UK wind resource: long term patterns and relationship to energy demand", Energy Policy vol. 35(1), 112-127.

[3] H. Zhang, J. Su, 2008, "Naïve Bayes for optimal ranking", Journal of Experimental and Theoretical Artificial Intelligence, vol. 20(2), 79-93.

[4] Ofgem, 2007, Renewables Obligation: Guidance for small generators $(50 \mathrm{~kW}$ or less), ref. 77/07. Ofgem, London, UK.

[5] European Union, 2004, Measuring Instruments Directive 2004/22/EC, Brussels, Belgium, 61-64.

[6] Ofgem, 2007, Renewables Obligation: Fuel Measurement and Sampling Guidance, ref. 57/07. Ofgem, London, UK, 75.

[7] W. Abrahamse, L. Steg, C. Vlek, T. Rothengatter, 2005. A review of intervention studies aimed at household energy consumption. Journal of Environmental Psychology, vol. 25, 273-291.

[8] Department of Communities and Local Government, 2007, Building a Greener Future: policy statement, DCLG publications, London, UK. 